\title{
Leveraging Mobility to Improve Quality of Service in Mobile Networks
}

\author{
Kimaya Sanzgiri Elizabeth M. Belding-Royer \\ Dept. of Computer Science \\ University of California \\ Santa Barbara, CA 93106 \\ \{kimaya, ebelding\}@cs.ucsb.edu
}

\begin{abstract}
Existing solutions for QoS-aware routing in mobile multi-hop networks attempt to discover a QoSsatisfactory route for the user at the user's current location. If a suitable route is not available at the current location, the user is denied access. This approach does not take advantage of the special characteristics of mobile multi-hop networks. That is, users in these networks are mobile and their connectivity is dependent on their location and the locations of other users. By changing their location, users can alter their connectivity characteristics and potentially obtain better service from the network. In this paper, we propose enhancing QoS-aware routing protocols to take advantage of this characteristic. With our solution, if a satisfactory route is not available at the user's current location, the protocol discovers a nearby location where a better route is available. The user can then choose to move to the suggested location to improve the received quality of service. We demonstrate our idea by appropriately extending a QoS-sensitive version of the AODV routing protocol, and presenting a simulation-based evaluation. Simulation results show the effectiveness and efficiency of our idea.
\end{abstract}

\section{Introduction}

The growing popularity of multimedia applications and the increasing support for multimedia content in various wireless computing devices have created a need for the network support of these applications in mobile ad hoc networks. Multimedia applications that involve streaming, or real-time continuous transfer, of multimedia data across the network are sensitive to end-to-end delay and jitter. To effectively support these applications, the network needs to ensure sufficient resource availability so that the applications receive the desired quality of service (QoS). This problem has been studied in great detail for wired networks and many solutions have been proposed $[3,4,12]$. However, the problem is significantly more difficult in mobile networks. Due to the shared nature of the wireless medium, the resources at each node are affected by the activities of others in its neighborhood. Also, mobility and dynamic topology cause the neighborhood of a node to continually change. These characteristics make it almost impossible to make any hard guarantees about resource availability and service quality in ad hoc networks. However, QoS-aware routing and admission control, together with better-than-best-effort packet delivery for high priority traffic [12], can significantly improve service.

Several researchers have proposed solutions for QoSaware routing and admission control in ad hoc networks $[1,6,7,10,18,19]$. Most of the proposed solutions approach the problem as follows. Given the QoS requirements of the application, the protocol queries the network to determine whether a satisfactory path currently exists. If a suitable path is found, it is made available to the application; otherwise the application is denied a route. This approach is similar to the IntServ technique used in wired networks [3]. However, it does not take advantage of the special characteristics of wireless networks. Unlike their wired counterparts, users in an ad hoc network are typically mobile. Also, their connectivity to the network is dependent on their location and the locations of other users, and is therefore not rigid. More specifically, users are capable of changing their location, and thereby their connectivity, to obtain better service from the network.

The idea of moving to a new location for better network service is often put to use by cellular phone users. In areas of poor connectivity, users often wander around with their phones, observing the display bars that indicate signal strength, in an attempt to find a location with 
sufficiently strong connectivity. We propose the application of the same idea for obtaining better QoS in ad hoc networks. However, instead of the user trying to guess where to move, we propose adding this intelligence to the network. With our proposed solution, if a route with the desired QoS constraints is not available to the user at their current location, the network suggests a nearby location where a better route is available. The user can then choose to move to the suggested location to improve the received quality of service.

The following example illustrates this idea. Consider a mobile network formed by students and faculty on a university campus. Suppose a faculty member needs to participate in an important on-line meeting. The students in a classroom near the faculty member's current location are heavily using the network. This causes the wireless medium to be congested, and so the faculty member is unable to obtain a path with the desired constraints. However, there is less network activity in the classrooms at the other end of the corridor, and so the medium at that location is relatively free. With our solution, the routing protocol discovers this fact and informs the faculty member, who then carries her laptop/PDA to an empty office down the corridor and is able to successfully participate in the on-line meeting. Thus, our solution not only improves the service experienced by the user, but also attempts to spatially redistribute users and make the overall usage of the wireless medium more efficient.

We note that this idea is not necessarily applicable to all ad hoc networks. For example, in an ad hoc network formed by cars moving on a freeway, the idea of moving to a specific location for better service does not make much sense, since the nodes are restricted to the traffic lanes and are continuously on the move in any case. However, we believe there are many scenarios where such a solution can be very useful. Ad hoc networks formed by students on a campus, users at a conference, geography researchers out in the field, etc., are some such example networks.

In this paper, we first describe a QoS-aware modification of the AODV routing protocol [15], called QoSAware AODV (Q-AODV). This serves as our base QoS routing protocol. The idea of leveraging mobility, which is our primary contribution, is then demonstrated by appropriately modifying Q-AODV ${ }^{1}$. We call the resulting protocol Mobility-Enhanced QoS-Aware AODV (MQAODV). Our evaluation of the protocol clearly demonstrates the benefits of our idea.

1 The idea of leveraging mobility is conceptually independent of the routing mechanism, and can therefore potentially be applied to any QoS routing scheme or implemented independent of the routing protocol.
The remainder of this paper is organized as follows. Section 2 briefly surveys related work. In section 3, we present a high-level overview of our proposed solution, before describing the details of Q-AODV and MQAODV in sections 4 and 5, respectively. A simulationbased evaluation of the protocols is presented in section 6. Finally, section 7 concludes the paper.

\section{Related Work}

Several QoS routing algorithms for mobile ad hoc networks have been proposed [1, 6, 7, 10, 18, 19]. We believe that most of these can be extended to leverage node mobility as described in this paper.

The QoS routing protocol proposed by Zhu and Corson [19] extends the route discovery phase of AODV for bandwidth computation, and is similar to the Q-AODV protocol described in this paper. However, it is designed to work in TDMA networks, and so the mechanism used for bandwidth computation is different. Both of these protocols loosely use the AODV QoS extension [14] to carry QoS information in the routing packets.

The idea of changing location to improve received quality of service was first presented in [16]. The solution envisioned by the author is specified for infrastructured wireless networks, and is outlined at a very high level, with no implementation details or experimental validation. It is based on a pervasive computing environment that gathers inputs from various layers of the system. Our solution is inspired by this vision. We address a narrower, more specific problem of moving within a mobile network to improve quality of service for multimedia applications. Further, we extend the idea presented in [16] to include multi-hop mobile networks.

Balachandran et. al. propose network-directed roaming to mitigate congestion hot-spots in public area wireless LANs [2]. Mobile users are directed to move from heavily loaded cells to less heavily loaded ones for improving service. This approach is targetted at infrastructured wireless networks. The computation is performed entirely by the infrastructure nodes (access points), who share their local information over the wired connection and collectively have complete knowledge about the network. Our approach, while similar in concept, is targetted at multi-hop mobile networks and is fully distributed. A mobile multi-hop network provides a far more challenging environment for such a solution, due to the unstructured and dynamic topology and lack of special nodes with complete knowledge about the network.

Several researchers have suggested using mobility to deliver messages in disconnected or intermittently connected ad hoc networks [11, 17]. In [11], sources and intermediate nodes are asked to explicitly change their 


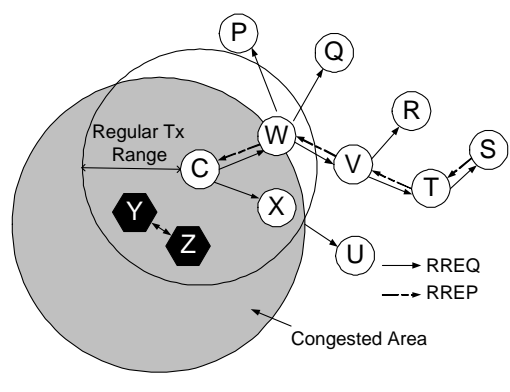

(a) AODV

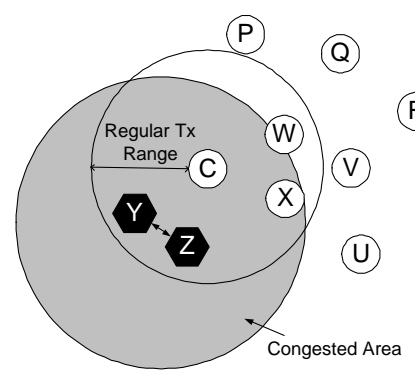

(b) Q-AODV
(R)
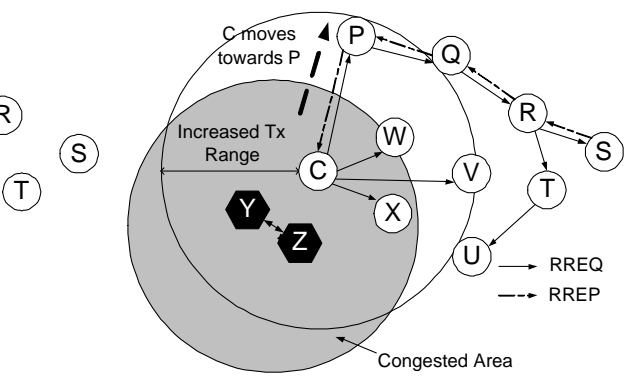

(c) MQ-AODV

Figure 1. Operation of AODV, Q-AODV and MQ-AODV in a sample network.

movement direction for message transmission. Requiring intermediate nodes to change their trajectory for message delivery is practical only in applications where all network nodes belong to the same authority and are working for a common cause. In our solution, a node changes its location only for its own benefit.

\section{Overview}

In this section, we present a high-level overview of our proposed solution. Details are given in the following sections.

As mentioned in section 1, the idea of leveraging node mobility to improve QoS can potentially be applied to any QoS-aware routing scheme. To demonstrate this idea, we chose to work with our modification of the AODV routing protocol, called Q-AODV, that enhances AODV to be aware of resource requirements.

In order to discover a route, AODV broadcasts a route request message (RREQ) throughout the network. When this message reaches the destination node, the destination sends a route reply (RREP) along the travelled path back to the source. AODV does not consider resource availability at nodes, and simply assumes that the best path is the one with the least number of hops. This does not necessarily work well for QoS-sensitive applications, as demonstrated by our experiments in section 6.

Consider the sample ad hoc network shown in figure 1 . The client $C$ needs to discover a route to the server $S$ with some QoS constraints so that it can receive a multimedia stream. Nodes $\mathrm{Y}$ and $\mathrm{Z}$ in the neighborhood are heavily using the medium, causing congestion in the surrounding area, colored gray in the figure. As a result, $\mathrm{C}$ does not have sufficient bandwidth available in its current location. As illustrated in figure 1(a), AODV does not consider resource availability while discovering a route. As a result, $\mathrm{C}$ obtains a route that passes through the congested area, which does not meet the bandwidth requirement.

Q-AODV enhances AODV to add QoS-sensitivity. It extends the AODV RREQ message to include information about resource requirements. Since bandwidth is a critical resource for multimedia applications, we focus on the discovery of paths satisfying the bandwidth requirements of the application. As the RREQ travels through the network, nodes forward it only if they have enough bandwidth to meet the specified requirement; otherwise, the RREQ is dropped. The RREQ that reaches the destination is guaranteed to have travelled along a path that has sufficient resources. If no such path is available, the RREQ will not reach the destination and the application will be denied a route. Section 4 describes Q-AODV in detail.

Figure 1(b) illustrates the operation of Q-AODV in the sample network. Since C does not have sufficient available bandwidth in its current location, it does not initiate an RREQ, and as a result, does not discover a route to the server.

To leverage node mobility, we further enhance QAODV as follows. While broadcasting the RREQ message, the source node increases its transmission power. This allows the RREQ to directly reach a larger set of nodes, some of which are not within the source's regular transmission range. These nodes all become potential candidates to directly offer the source the route it needs. If the best route is offered by a node that is not within the source's regular transmission range, the source can choose to move towards that node to use the route. We call this modified protocol Mobility-Enhanced QoS-Aware AODV (MQ-AODV). A detailed description is presented in section 5 .

Referring to figure 1(c), we see that using MQAODV, $\mathrm{C}$ broadcasts the RREQ with increased trans- 
mission power, causing it to be directly received by node $\mathrm{P}$, which is not within $\mathrm{C}$ 's regular transmission range. Node $\mathrm{P}$ is outside the congested area, and therefore able to offer $\mathrm{C}$ a satisfactory route. MQ-AODV discovers and reports this information to $\mathrm{C}$, who can then choose to move closer to $\mathrm{P}$ and use the offered route.

Note that we have used the disk model, i.e. perfect circles, to represent transmission ranges in the figure. In real environments, this might not be the case because of various factors such as multipath interference, obstacles, etc. A circular transmission range is not critical to the validity of our idea and the operation of our proposed protocol. We further note that even if the transmission range is not circular, an increase in transmission power will result in an increased range in most cases. For the remainder of the paper, we assume circular ranges in order to simplify the description of our approach.

\section{QoS-Aware AODV}

In this section, we describe the details of Q-AODV. Our modifications only affect the Route Discovery phase of AODV; the Route Maintenance phase remains unmodified.

Q-AODV is a stateless protocol. Resource availability is only checked during flow admission. Resource availability can change as nodes move, and is therefore not guaranteed. For this reason, no resource reservations are made. We do not perform any flow policing and assume that flows are well-behaved. Flow monitoring and adjustment of flow rate is also left to the application.

\subsection{Route Discovery with QoS Constraints}

We extend the AODV Route Request (RREQ) packet to include the following fields: minimum bandwidth, maximum bandwidth and bottleneck bandwidth. The first two fields specify the bandwidth requirements of the application and are populated by the source node. The AODV RREQ flooding procedure is then followed, with one modification: a node forwards the RREQ only if it has enough bandwidth available to satisfy the request. Bandwidth availability at each node is determined by the procedure described in section 4.2. The bottleneck bandwidth field contains the least bandwidth encountered along the path, and is appropriately populated by nodes forwarding the RREQ.

On receiving the RREQ, the destination replies with a Route Reply (RREP). The RREP is extended to include a field specifying the available bandwidth on the path (this is obtained from the bottleneck bandwidth field in the RREQ). The RREP is then forwarded back to the source as in unmodified AODV with no additional processing at intermediate nodes.

To ensure that sufficient bandwidth is available on the end-to-end path, the RREQ must traverse the complete route to the destination. For this reason, no intermediate node is allowed to reply to the RREQ; only the destination may respond. Also, traffic from different sources to the same destination may need to be forwarded along different paths based on session requirements and resource availability. For this reason, Q-AODV discovers and maintains routes on a per-source-destination-pair basis rather than a per-destination basis as in AODV.

In Q-AODV, unlike AODV, it is beneficial for the destination to reply to multiple RREQ instances, since the first RREQ instance to reach the destination need not have travelled along the most resourceful path; another path with higher bandwidth availability may be present. The source can then select the best path from among the RREPs received. After selecting the best path, the source informs the destination of its decision by sending a message along the path, so that the destination can use the same path for reverse communication.

We note that Q-AODV still has room for improvement. Q-AODV does not consider contention among multiple nodes on a path, a network characteristic described in $[6,18]$. Also, if multiple sessions attempt a route discovery simultaneously, they may all be admitted even though the available resources are insufficient to support their aggregated requirements. These shortcomings are also present in other proposed QoS routing protocols, such as $[1,19]$.

\subsection{Available Bandwidth Determination}

In order to find a path that satisfies the application's bandwidth requirements, we must first determine the bandwidth availability at each node. Determining available bandwidth at a wireless node is a non-trivial task, especially due to mobility and the shared wireless medium. Available bandwidth at a node is affected not only by its own transmissions, but also the transmissions of the nodes in its vicinity.

We use the mechanism presented in [6] to determine bandwidth availability at a node. A brief description of this mechanism follows. The reception range of a node is the maximum separation between a sender and receiver for successful reception of packets. Further, the receiver interference distance is defined as the minimum distance between a receiver and another sender, such that the sender's transmissions do not interfere with the receiver's ability to successfully receive packets from its own sender. The reception range and receiver interference distance are denoted by $R_{x}$ and $R I D$, respectively. 


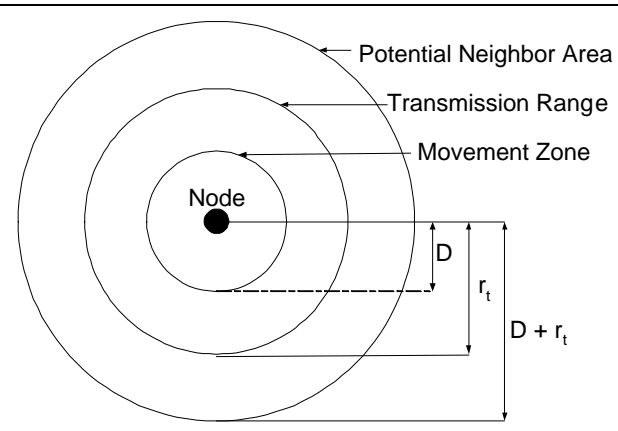

Figure 2. Relation between movement zone, transmission range and location of potential neighbors.

The authors of [6] observe that the minimum distance between two wireless nodes for successful simultaneous transmissions is $\left(R_{x}+R I D\right)$. Further, if acknowledgements are used at the MAC layer, the minimum distance increases to $\left(2 * R_{x}+R I D\right)$, in order to prevent collisions of acknowledgements (note that, according to the IEEE 802.11 specification [9], no medium sensing is performed before transmitting an acknowledgement). Thus, a wireless node can transmit if no other node within $\left(2 * R_{x}+R I D\right)$ range is simultaneously transmitting.

By adjusting its carrier sensing range to $\left(2 * R_{x}+\right.$ RID), a node can sense the transmissions of the other nodes relevant to bandwidth calculation. The node then senses the medium to determine the fraction of time that the medium is idle. This fraction, along with the maximum available bandwidth, indicates the current bandwidth availability. Further details about this approach can be found in [6].

\section{Mobility-Enhanced QoS-Aware AODV}

We now describe how Q-AODV can be extended to leverage node mobility, as indicated in section 1. We call this protocol Mobility-Enhanced QoS-Aware AODV (MQ-AODV). The protocol requires that all nodes know their geographical coordinates, either through GPS or some other location system. We also assume that all nodes have a uniform default transmission range, but are capable of increasing their transmission power to reach a larger range if necessary. We do not consider the presence of obstacles.

\subsection{Modified Route Discovery}

To begin, the user must indicate to the routing protocol that she is willing to move to a different location if necessary for obtaining the desired quality of service. The user can also specify the area within which she is willing to move; otherwise a default is assumed. We call this the movement zone of the user. In this paper, we assume that the user is prepared to move in any direction for a maximum specified distance. In other words, the movement zone is a circular region centered at the user, as shown in figure 2. More complex movement zones can be handled by a straightforward extension to the protocol.

By moving to different locations in the movement zone, the node can potentially have different neighbor sets. The union of all such neighbor sets forms the potential neighbor set for the node with respect to the movement zone. If the movement zone is a circular region of radius $D$ and the transmission range of the node is $r_{t}$, then the maximum distance of a potential neighbor from the node's current location is $\left(D+r_{t}\right)$. In other words, all potential neighbors are located within a circular area of radius $\left(D+r_{t}\right)$ centered at the node, as shown in figure 2.

Once the movement zone is known, MQ-AODV initiates route discovery by broadcasting the RREQ packet at a higher transmission power, such that it reaches all potential neighbors. The transmission power to be used is determined from the maximum distance to a potential neighbor. The RREQ packet is extended to include the user's location coordinates, in addition to the information added by Q-AODV as described in section 4.1.

On receiving the RREQ packet, potential neighbors record the location of the RREQ source. The rest of the route discovery procedure is the same as Q-AODV. Intermediate nodes broadcast the RREQ packet at regular transmission power.

When the destination receives the RREQ, it replies with an RREP. The RREP processing is also similar to Q-AODV. However, before forwarding the RREP, each node checks to see whether it is the last hop on the route, i.e., whether it is forwarding the RREP directly to the source. The last-hop node belongs to the source's potential neighbor set. If a node is the last hop, it looks up the location information of the source, which it had saved when it received the RREQ. From this information, it determines its distance from the source. If this distance is greater than the regular transmission range, the node increases its transmission power sufficiently before forwarding the RREP. The RREP packet is extended to include the location information of the last hop.

On receiving the RREP, the source inspects the lo- 


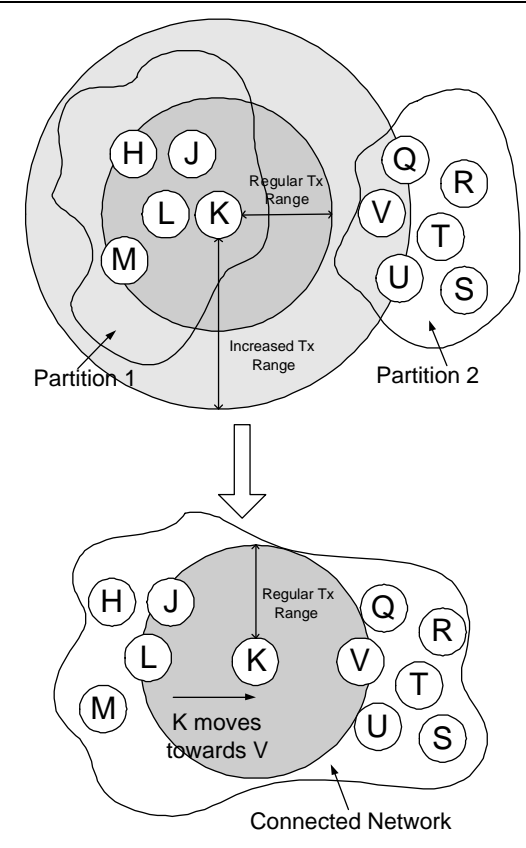

Figure 3. Partitioned network scenario.

cation information of the last hop. If this location is within the node's regular transmission range, then the node does not need to move and can immediately use the route. If it is outside the transmission range, the user is notified, who can move in the direction of the potential neighbor until it is within range ${ }^{2}$. If multiple RREPs are sent, as mentioned in section 4.1, the user can wait for some timeout period to receive them before deciding whether she needs to move and in what direction.

Some routing protocols, such as AODV, invalidate a route if it remains unused for a certain time limit. If the time taken by the user to move to the new location is greater than this time limit, the route may be invalidated. In such a case, the user should repeat the route discovery process at the new location, without increasing transmission power, to refresh the route.

\subsection{Connectivity in Disconnected Networks}

An important benefit of MQ-AODV is that it can help users gain connectivity to disconnected parts of the network. For example, consider the partitioned network shown in figure 3. Using regular transmission power, nodes in one partition cannot reach the nodes in the other

2 We assume that the user is capable of determining the required direction of motion from the geographical coordinates of the potential neighbor. The software can assist the user in this task by pointing out the location on a map. partition, and are disconnected from each other. With MQ-AODV, nodes transmit route requests at a higher transmission power, and may therefore be able to reach nodes in the other partition. In the figure, node $\mathrm{K}$ is able to reach node $\mathrm{V}$ by increasing its transmission power. Further, once a user establishes a route to a node in the other partition, the user moves towards that node and can then act as a bridge connecting the two partitions. As seen in the figure, node $\mathrm{K}$ moves towards node $\mathrm{V}$, and can then relay traffic between the two partitions, thus connecting them. AODV and Q-AODV, on the other hand, do not have this benefit.

\subsection{Limitations}

MQ-AODV has some limitations. First, if the user is currently located a very short distance away from nodes that are causing congestion in the medium, she may need to move a significantly large distance in order to exit the congested region. For this, a large movement zone needs to be specified, which correspondingly increases the transmission power required to reach all potential neighbors. Since power varies hyperbolically with distance, with exponent between two and four, this high power transmission may become too expensive beyond a certain distance, thus limiting the size of the movement zone. Our experiments, presented in section 6, demonstrate that even with a limited movement zone of radius 150m, MQ-AODV improves performance over QAODV.

Second, if multiple users perform route discovery with MQ-AODV simultaneously, they may all be directed to move toward the same location. This location may be incapable of supporting the aggregated resource requirements of multiple users. If the probability of multiple users performing route discovery simultaneously is low, this limitation is not significant. If the probability is high, MQ-AODV needs to be enhanced to address this problem. This is part of our future work.

\section{Protocol Evaluation}

We evaluate our idea of leveraging node mobility for improving quality of service by comparing the performance of AODV, Q-AODV and MQ-AODV through simulation. The NS-2 [8] simulator is used for the evaluation. The mobility-enhanced and QoS-aware versions of AODV are built on top of the AODV-UU implementation for NS-2 [13]. In the following sections, we first present our application model, followed by experiment details and results. 


\subsection{Application Model}

We model a simple multimedia application by appropriately modifying the CBR application in NS-2. Our application is a client-server-based streaming media application. The server streams media, such as music and movies, over the network to the client at the client's request. The application session is initiated by the client. The client first discovers a route to the server ${ }^{3}$, and then requests the server to begin streaming the data. If no route with sufficient available bandwidth is found, the client backs off for a random interval of between 10 and 20 seconds and then re-attempts to discover a suitable route.

The server begins streaming data to the client once it receives the client's request. If, during the session, the route to the client is lost and cannot be re-established, the server pauses its transmission and re-attempts to discover a route after a random interval of between 10 and 20 seconds.

\subsection{Experiment Setup}

We run simulations of 50 nodes randomly placed in a $1000 \mathrm{~m} \times 1000 \mathrm{~m}$ area. Each run is executed for $500 \mathrm{sec}-$ onds of simulation time. The Two Ray Ground propagation model is used, and the transmission range and carrier sensing range of nodes is set to $250 \mathrm{~m}$ and $550 \mathrm{~m}$, respectively. IEEE 802.11 is used as the MAC protocol.

For MQ-AODV, the movement zone is considered to be a circular region of radius $150 \mathrm{~m}$ centered at the source node. Only the nodes running the application client can move to improve quality of service, and they can do this at most once. It may be reasonable to expect users to change their location multiple times to improve service in some scenarios, but we avoid this in the simulations.

The mobility pattern of nodes is as follows: 20 nodes are static while 30 nodes move following the randomwaypoint mobility model [5]. The pause time is $20 \mathrm{sec}-$ onds. Of the 30 mobile nodes, 10 nodes move at random speeds up to $5 \mathrm{~m} / \mathrm{s}, 10$ nodes at random speeds between 5 to $10 \mathrm{~m} / \mathrm{s}$, while the remaining 10 nodes move at random speeds between 10 to $20 \mathrm{~m} / \mathrm{s}$. We believe that this mobility pattern, which we call Graded Mobility, is characteristic of a deployment scenario such as a campus, where some users are stationary, some are walking, while others are riding bicycles or driving. The client and server nodes for the application are picked out of

3 The QoS path discovered by Q-AODV and MQ-AODV is bidirectional, and so the client can do the QoS route discovery even though the data will be sent by the server. the 20 static nodes. It is a reasonable assumption that clients are static, except when they change their location to improve quality of service. Moving to a new location for better quality of service does not make sense unless the node is going to remain stationary after reaching the new location. Also, in typical deployment scenarios, the server nodes are likely to be nodes in the wired internet. In this case, from the perspective of the admission control protocol, the gateway between the wired and wireless networks is treated as the server, and it is therefore stationary as well. As mentioned in section 1, our solution is not necessarily applicable to highly mobile networks. However, there are a large number of deployment environments with low to medium mobility, which can benefit from such a solution. The Graded Mobility pattern is representative of such environments.

Between two and ten application sessions are started 10 seconds apart in each simulation run. In each session, the server streams data at $64 \mathrm{kbps}$ for 180 seconds of execution (excluding pauses). The size of each data packet is 512 bytes, so approximately 16 packets are transmitted per second.

For simplicity, the destination node only replies to the first RREQ instance in our simulations for both QAODV and MQ-AODV.

\subsection{Performance Metrics}

We measure the following performance metrics to evaluate the effectiveness and efficiency of the protocol:

- Packet delivery fraction: This is the fraction of data packets sent by the server that are received by the client. The higher the packet delivery fraction, the more effective the protocol in reducing network congestion and the better the user experience in viewing/hearing the media stream.

- Number of data packets received: This is the total number of data packets received by the clients. It indicates the data throughput obtained. The packet delivery fraction alone is not enough to indicate the effectiveness of the protocol in delivering packets, since the number of packets sent with each routing protocol may be different (the QoS routing protocols do not admit a session unless a route with the required QoS is available). This metric therefore complements the packet delivery fraction in determining protocol effectiveness.

- Average end-to-end packet delay: This is the average end-to-end delay of packets that are received by the client. As this value decreases, the congestion in the network is reduced, and packets are more likely to be received in time for playout. 
- Fraction of received packets with unacceptable end-to-end delay: Many multimedia applications, such as Internet telephony and live media broadcast, place an upper limit on acceptable end-to-end packet delay. For example, in typical voice applications, packets with end-to-end delay greater than 400 milliseconds arrive too late to be played out. Such packets waste the resources of the network since they traverse the path between the source and destination but are received too late to be used by the destination. This metric measures the fraction of received packets that are thus unacceptably delayed. A low value for this fraction indicates more efficient usage of network resources and better quality perceived by the user.

- Routing load: This is the number of routing control bytes transmitted per data byte received by the application client. Each transmission of a control packet by intermediate nodes is counted as one transmission. This metric indicates the overhead imposed by the routing protocol on the network.

\subsection{Experiment Results}

Figure 4 presents results from experiments. Each metric is plotted against the total number of data sessions created ${ }^{4}$. Each data point represents an average over ten simulation runs with different randomlygenerated mobility.

While comparing the performance of AODV to the QoS-sensitive protocols, it is important to note that AODV does not perform admission control and hence all data sessions created are immediately admitted into the network. Q-AODV and MQ-AODV, on the other hand, delay the admission of sessions until sufficient resources are available. The performance of the protocols is thus not directly comparable. However, we plot the performance of AODV in the graphs in order to emphasize the importance of admission control.

Figures 4(a) and 4(b) show the packet delivery fraction (PDF) and the total number of data packets received, respectively. Using AODV, the PDF drops significantly as the traffic increases. This is due to the absence of admission control, leading to congestion in the network. With QoS-aware routing, the PDF remains roughly constant even as the number of sessions increase. This is achieved by delaying the admission of sessions until sufficient bandwidth is available. As seen in the figure, the

4 The number of data sessions created may be different from the number of data sessions admitted in the case of Q-AODV and MQ-AODV, since these protocols perform admission control.
PDF is roughly $90 \%$ when using Q-AODV, and rises to above $95 \%$ with MQ-AODV. When clients change their location, they tend to move away from locations where bandwidth usage is high, and often move closer to the server, reducing the number of hops on the route and resulting in fewer link breaks and packet drops. For similar reasons, more data packets are delivered to their destinations with MQ-AODV as compared to Q-AODV, as shown in figure 4(b). Although AODV also delivers a large number of data packets, its packet delivery fraction indicates that these packets are a small fraction of those sent. Since AODV is not QoS-sensitive, it admits all data sessions, resulting in a much larger number of data packets sent. Although Q-AODV and MQ-AODV admit fewer sessions, the number of data packets delivered is comparable to AODV, and greater in case of MQAODV.

The average end-to-end delay of received packets is shown in figure 4(c), while figure 4(d) shows the fraction of received packets whose end-to-end delay exceeds $400 \mathrm{~ms}$. MQ-AODV decreases both the average delay and the fraction of packets that are unacceptably delayed as compared to Q-AODV. This is again due to reduced congestion and decreased route lengths. For similar reasons, MQ-AODV's routing load is lower than that of QAODV, as seen in figure 4(e). Both protocols clearly outperform AODV in all metrics.

Our results thus indicate that both Q-AODV and MQAODV improve the received QoS over AODV. MQAODV provides additional benefits over Q-AODV, such as increased packet delivery fraction, decreased end-toend delay and lower response time, while simultaneously reducing the routing load in the network.

In addition to these tests, we ran an experiment simulating the scenario described in section 5.2 and verified that it indeed works as expected. Figure 4(f) shows the result from this experiment. As seen in the figure, all data sessions are admitted using MQ-AODV, while none are admitted when using Q-AODV or AODV.

Finally, to further stress our solution, we also ran experiments where only the client nodes are stationary; all other nodes move at random speeds between 0 and 20 $\mathrm{m} / \mathrm{s}$. The results of these experiments, omitted for lack of space, also show that MQ-AODV improves performance even in this scenario.

\section{Conclusion}

In this paper, we have investigated how node mobility can be leveraged to improve quality of service in mobile networks. The proposed protocol, MQ-AODV, demonstrates this idea by enhancing Q-AODV, a QoS-sensitive version of the AODV routing protocol. Simulation re- 


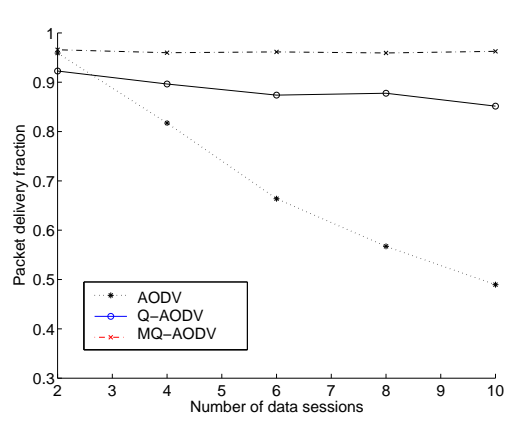

(a) Packet delivery fraction

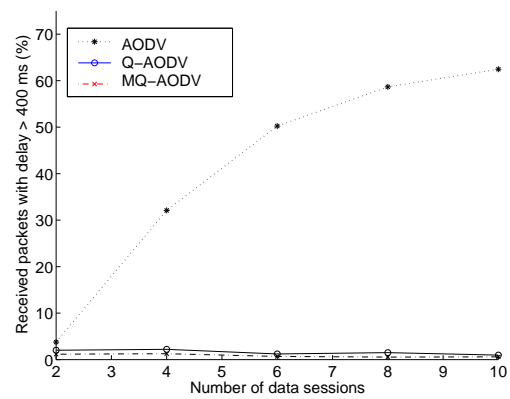

(d) Fraction of received packets with delay greater than $400 \mathrm{~ms}$

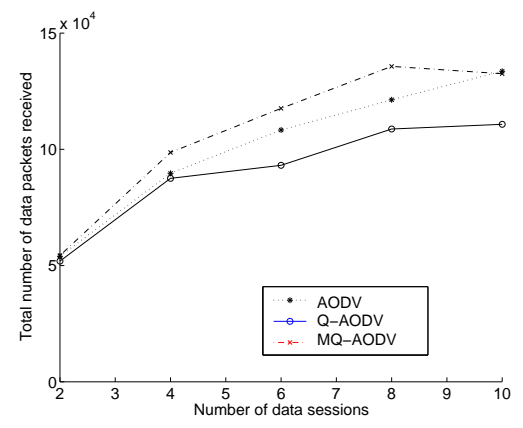

(b) Number of data packets received

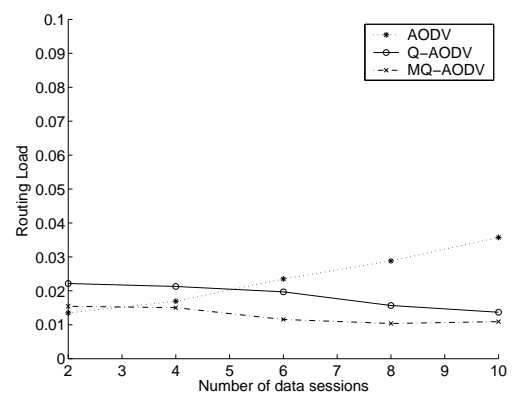

(e) Routing load

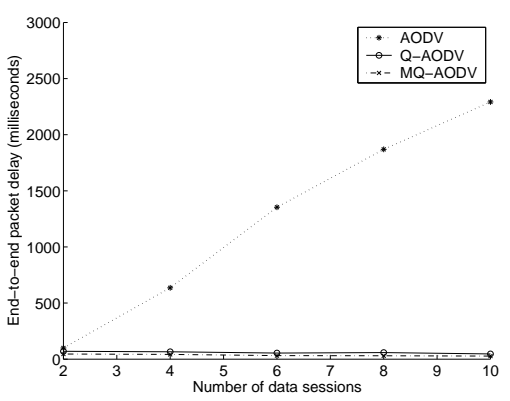

(c) End-to-end delay

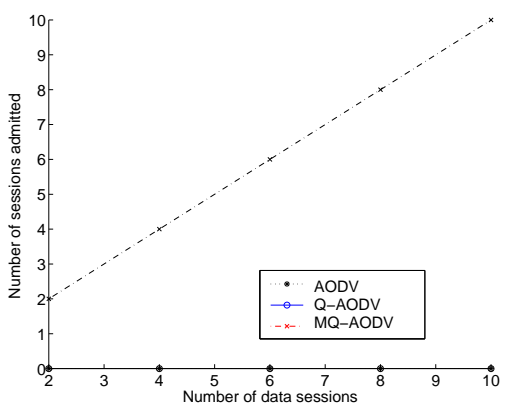

(f) Number of sessions admitted in the partitioned network scenario

Figure 4. Performance results.

sults show that the idea is indeed effective in mobile ad hoc networks with low to medium mobility, and can be accomplished with low overhead.

In the future, we plan to examine how other QoSaware routing protocols can be extended to use this idea. We will also look at how this approach can be made to work in the presence of obstacles. With obstacles, some potential neighbors may be unable to directly receive the high power broadcast, and so a multi-hop mechanism may be necessary.

Some limitations of MQ-AODV were described in section 5.3. Our future work includes addressing these limitations. In particular, we plan to remove the restriction imposed by the maximum transmission range and design a solution that enables a mobile node to look beyond a single hop to find a location with better connectivity. Finally, the idea of leveraging mobility to improve service can also be extended to intermediate nodes on a network path in some deployment scenarios. This would make sense primarily in scenarios where all network nodes are owned by a common authority and have a common goal.
Acknowledgement: This research is supported by NSF Infrastructure grant EIA-0080134 and by a grant from the UC-Micro program with Nokia Corporation.

\section{References}

[1] G. Ahn, A. Campbell, A. Veres, and L. Sun. SWAN: Service Differentiation in Stateless Wireless Ad Hoc Networks. In IEEE Infocom, 2002.

[2] A. Balachandran, P. Bahl, and G. Voelker. Hot-Spot Congestion Relief in Public-area Wireless Networks. In IEEE WMCSA, 2002.

[3] R. Braden, D. Clark, and S. Shenker. Integrated Services in the Internet Architecture: an Overview. RFC 1633, June 1994.

[4] R. Braden, L. Zhang, S. Berson, S. Herzog, and S. Jamin. Resource Reservation Protocol (RSVP) - Version 1 Functional Specifi cation. RFC 2205, September 1997.

[5] J. Broch, D. Maltz, D. Johnson, Y.-C. Hu, , and J. Jetcheva. A Performance Comparison of Multi-hop Wireless Ad Hoc Network Routing Protocols. In ACM MobiCom, 1998. 
[6] I. Chakeres and E. Belding-Royer. PAC: Perceptive Admission Control for Mobile Wireless Networks. Technical Report 2004-17, University of California, Santa Barbara, June 2004.

[7] T. Chen, J. Tsai, and M. Gerla. QoS Routing Performance in Multihop Multimedia Wireless Networks. In IEEE ICUPC, 1997.

[8] K. Fall and K. Varadhan. The NS Manual. http://www.isi.edu/nsnam/ns/doc/.

[9] IEEE Computer Society. IEEE 802.11 Standard, IEEE Standard For Information Technology, 1999.

[10] S.-B. Lee, G.-S. Ahn, X. Zhang, and A. Campbell. INSIGNIA: An IP-based Quality of Service Framework for Mobile Ad hoc Networks. Journal of Parallel and Distributed Computing, 60(4):374-406, 2000.

[11] Q. Li and D. Rus. Sending Messages to Mobile Users in Disconnected Ad-hoc Wireless Networks. In ACM MobiCom, 2000.

[12] K. Nichols, S. Blake, F. Baker, and D. Black. Defi nition of the Differentiated Services Field (DS Field) in the IPv4 and IPv6 Headers. RFC 2474, December 1998.

[13] E. Nordstrom and B. Wiberg. The AODV-UU Implementation. http://www.docs.uu.se/scanet/aodv.

[14] C. Perkins and E. Belding-Royer. Quality of Service for Ad-hoc On-demand Distance Vector Routing. IETF Internet Draft, draft-perkins-manet-aodvqos-02.txt, October 2003. (Work in progress).

[15] C. Perkins and E. Royer. Ad-hoc On-Demand Distance Vector Routing. In IEEE WMCSA, 1999.

[16] M. Satyanarayanan. Pervasive Computing: Visions and Challenges. IEEE Personal Communications, August 2001.

[17] A. Vahdat and D. Becker. Epidemic Routing for Partially Connected Ad hoc Networks. Technical Report CS-2000-06, University of California, San Diego, July 2000.

[18] Y. Yang and R. Kravets. Contention-Aware Admission Control for Ad Hoc Networks. Technical Report 20032337, University of Illinois at Urbana-Champaign, April 2003.

[19] C. Zhu and S. Corson. QoS Routing for Mobile Ad Hoc Networks. In IEEE Infocom, 2002. 